\title{
Stabilin-1-Mediated Efferocytosis Protects against Vascular Leakage in Sepsis: A Novel Therapeutic Approach?
}

\author{
Peter Mirtschink $^{1} \quad$ Triantafyllos Chavakis $^{1}$ \\ ${ }^{1}$ Institute of Clinical Chemistry and Laboratory Medicine, Technische \\ Universität Dresden, Dresden, Germany \\ Thromb Haemost 2018;118:1852-1853.
}

Sepsis is a complex clinical syndrome with high lethality characterized by systemic inflammation and organ failure. ${ }^{1}$ Although intensive research over the past decades has identified several mechanisms contributing to sepsis pathology, sepsis represents a major therapeutic burden. Current therapeutic strategies are limited to rapid initiation of antibiotic therapy and further supportive treatment, including fluids, the use of vasopressors and functional organ replacement. ${ }^{2}$ Therefore, sepsis was recently identified by the World Health Organization as a global health priority. ${ }^{3}$

In the context of sepsis pathogenesis and progression, an important mechanism is vascular leakage due to disruption of the vascular barrier by inflammatory stimuli. ${ }^{4,5}$ Maintenance of vascular integrity is supported by the efficient removal of apoptotic endothelial cells. The phagocytic clearance of damaged cells by macrophages, a process termed efferocytosis, induces resolution of inflammation and suppression of pro-inflammatory cytokines. ${ }^{6,7}$

In the previous issue of Thrombosis and Haemostasis, Lee et $\mathrm{al}^{8}$ proposed a new mechanism, by which macrophage Stabilin-1 (STAB-1), a phagocytic receptor mediating efferocytosis by recognizing phosphatidylserine on apoptotic cells, ${ }^{9}$ promotes the clearance of apoptotic vascular endothelial cells damaged by severe inflammation. This function of STAB-1 protects against disruption of vascular integrity in the course of sepsis. ${ }^{8}$ Low $\mathrm{pH}$ values, which are present in patients with septic shock, promote the macrophage expression of STAB- $1 .{ }^{9}$ Genetic deletion of STAB- 1 decreased the survival of septic mice in the model of cecal ligation and puncture. Decreased sepsis survival in STAB-1 deficiency was associated with diminished efferocytosis, increased vascular permeability and enhanced organ dysfunction. Interestingly, the pro-inflammatory mediator high-mobility group box 1 (HMGB1) ${ }^{10-12}$ inhibited STAB1-dependent efferocytosis of apoptotic cells. Consistently,
Address for correspondence Peter Mirtschink, MD, Institute of Clinical Chemistry and Laboratory Medicine, Technische Universität Dresden, Dresden, Germany (e-mail: peter.mirtschink@uniklinikum-dresden.de).

blockade of HMGB1 with a neutralizing antibody improved the phagocytic capacity of macrophages and reduced sepsis mortality.

Previous work by Palani et $\mathrm{a}^{13}$ demonstrated that STAB1 on monocytes suppresses the activation of Th1 lymphocytes; thus, STAB-1 may also exert an immunosuppressive action. In addition, STAB-1 may regulate lymphocyte migration and inflammatory cell recruitment. ${ }^{14}$ How the sepsisprotective action of STAB-1, as shown by Lee et al in this issue of Thrombosis and Haemostasis, ${ }^{8}$ may reconcile with its previously described immunomodulatory functions, requires future investigation. Thus, a therapeutic approach aiming at increasing STAB- 1 function needs to be carefully evaluated.

Taken together, the study by Lee et al demonstrated the important role of macrophage STAB-1 in protecting against vascular barrier dysfunction in sepsis via mediating enhanced efferocytosis of apoptotic endothelium. ${ }^{8}$ Testing the proposed sepsis-protective mechanism of STAB-1 merits further experimental and pre-clinical assessment.

Conflict of Interest

None.

\section{References}

1 Lelubre C, Vincent J-L. Mechanisms and treatment of organ failure in sepsis. Nat Rev Nephrol 2018;14(07):417-427

2 Rhodes A, Evans LE, Alhazzani W, et al. Surviving Sepsis Campaign: International Guidelines for Management of Sepsis and Septic Shock: 2016. Intensive Care Med 2017;43(03):304-377

3 Reinhart K, Daniels R, Kissoon N, Machado FR, Schachter RD, Finfer S. Recognizing sepsis as a global health priority - a WHO resolution. N Engl J Med 2017;377(05):414-417

4 Soon ASC, Chua JW, Becker DL. Connexins in endothelial barrier function - novel therapeutic targets countering vascular hyperpermeability. Thromb Haemost 2016;116(05):852-867 received

September 20, 2018

accepted

September 20, 2018 (c) 2018 Georg Thieme Verlag KG Stuttgart · New York
DOI https://doi.org/ $10.1055 / \mathrm{s}-0038-1673688$. ISSN 0340-6245. 
5 Russell JA, Rush B, Boyd J. Pathophysiology of Septic Shock. Crit Care Clin 2018;34(01):43-61

6 Kourtzelis I, Mitroulis I, von Renesse J, Hajishengallis G, Chavakis T. From leukocyte recruitment to resolution of inflammation: the cardinal role of integrins. J Leukoc Biol 2017;102(03):677-683

7 Fullerton JN, O'Brien AJ, Gilroy DW. Pathways mediating resolution of inflammation: when enough is too much. J Pathol 2013; 231(01):8-20

8 Lee W, Park S-Y, Yoo Y, et al. Macrophagic Stabilin-1 restored disruption of vascular integrity caused by sepsis. Thromb Haemost 2018;118(10):1776-1789

9 Park S-Y, Bae D-J, Kim M-J, Piao ML, Kim IS. Extracellular low pH modulates phosphatidylserine-dependent phagocytosis in macrophages by increasing stabilin-1 expression. J Biol Chem 2012;287(14):11261-11271
10 Lee W, Ku S-K, Bae J-S. Factor Xa inhibits HMGB1-induced septic responses in human umbilical vein endothelial cells and in mice. Thromb Haemost 2014;112(04):757-769

11 Orlova VV, Choi EY, Xie C, et al. A novel pathway of HMGB1-mediated inflammatory cell recruitment that requires Mac-1-integrin. EMBOJ 2007;26(04):1129-1139

12 Lotze MT, Tracey KJ. High-mobility group box 1 protein (HMGB1): nuclear weapon in the immune arsenal. Nat Rev Immunol 2005;5 (04):331-342

13 Palani S, Elima K, Ekholm E, Jalkanen S, Salmi M. Monocyte Stabilin-1 suppresses the activation of Th1 lymphocytes. J Immunol 2016;196(01):115-123

14 Karikoski M, Irjala H, Maksimow M, et al. Clever-1/Stabilin-1 regulates lymphocyte migration within lymphatics and leukocyte entrance to sites of inflammation. Eur J Immunol 2009;39(12):3477-3487 